\title{
Estrategia de operaciones, tecnología y rendimiento de operaciones en la industria hondureña: propuesta de un modelo universal
}

César H. Ortega Jiménez*

\section{RESUMEN}

El presente estudio establece algunos lineamientos para investigar cómo las fábricas productivas implementan la estrategia de operaciones y la tecnología, con el propósito de ampliar el entendimiento de la Dirección/Gestión de Producción y Operaciones (DGPO). Para ello, se propone testar individualmente la eficacia de ambas Prácticas Avanzadas de Producción (PAP), al establecer algunos de sus aspectos con el objetivo primordial de aumentar el rendimiento de operaciones. En relación a dicho objetivo, se identifican dos áreas de interés particular: 1) el posible impacto de la tecnología sobre el rendimiento de operaciones y 2) la posible influencia de la estrategia de operaciones sobre el rendimiento de operaciones. El presente estudio propone un modelo de ajuste universal para verificar ambas áreas y la metodología para recoger los datos de distintos sectores industriales en Honduras. Los resultados empíricos serán de vital importancia en la DGPO en Honduras ya que podrán sugerir si existen diferencias entre los niveles de implementación de ambas PAP, analizadas de forma individual e implementadas por dos tipos de fábricas: las de clase mundial (alto rendimiento) y las de rendimiento estándar.

Palabras clave: Estrategia de Operaciones; Tecnología; Rendimiento de Operaciones; Ajuste Universal.

\section{ABSTRACT}

The general objective of this paper is to investigate how manufacturing plants make use of manufacturing strategy and technology, aiming to increase the understanding of Production and Operations Management (POM). This is done by individually testing the effectiveness of both manufacturing practices (MP) by using some of their aspects, with the ultimate goal of enhancing operational performance. In keeping with the overall objective of this research, two areas are identified as being of

\footnotetext{
* Cesar H. Ortega Jimenez, cesortjim@alum.us.es

Facultad de Ciencias Económicas, Instituto de Investigaciones Económicas y Sociales,

Universidad Nacional Autónoma de Honduras.
} 
particular interest: (i) whether manufacturing strategy influences operational performance and (ii) whether technology influences operational performance. This paper proposes a model of universal fit to verify both areas, and a methodology for collecting the data from different industrial sectors in Honduras. The empirical results will be of vital importance in Honduras' $\mathrm{POM}$ since they will suggest if there are differences between the levels of both MPs being implemented by both high and standard performers.

Key words: Manufacturing Strategy; Technology; Operational Performance; Universal Fit. 


\section{INTRODUCCIÓN}

El modelo teórico propuesto del proyecto internacional de investigación Producción de Alto Rendimiento (High Performance Manufacturing, HPM) que sustenta el proyecto HPM hondureño y sirve para nuestro estudio se ha extendido de las seis áreas de prácticas de las dos primeras rondas realizadas (Schroeder y Flynn, 2001) a nueve, a saber, Estrategia de Operaciones, Gestión de Calidad (Total Quality Management, TQM), Justo a Tiempo (Just In Time, JIT) y Teoría de las Restricciones (Theory of Constrains, TOC), Recursos Humanos (RRHH), Tecnología de Información y Comunicaciones (TIC), Desarrollo de Nuevos Productos (New Product Development, NPD), Gestión de la Cadena de Suministro (Supply Chain Managemnt, SCM), Tecnología, Mantenimiento Productivo Total (Total Productive Maintenance, TPM).

Puede decirse que HPM es un conjunto integrado de procesos diseñados para alcanzar una ventaja competitiva mundial sostenible por medio de la mejora continua de la capacidad de producción (Schroeder y Flynn, 2001). Como modelo de producción dinámico que es, recoge en cada momento del tiempo, las mejores prácticas de fabricación para su posible inclusión como parte de los procesos de producción, dependiendo de la contingencia particular de la fábrica y de la integración de las nuevas prácticas a lo que la fábrica está haciendo o planea hacer.

Así pues, la idea que sustenta HPM es que las firmas productivas tienes caminos singulares hacia el alto rendimiento, basándose en los factores contingenciales y en la interconexión de las prácticas avanzadas de producción (PAP). Estudios anteriores sobre este tema arrojan poca luz sobre las razones que justifican que la aplicación de las mismas prácticas avanzadas de producción fomente alto rendimiento en unas fábricas, pero no lo haga en otras.

Sin embargo, en la práctica, lograr ventaja competitiva sostenible por medio de HPM es en sí un "objetivo evasivo": algunas veces se encuentran fábricas con alto rendimiento y con prácticas relativamente pobres. Bien es cierto que, en este caso, el rendimiento de la fábrica puede disminuir rápidamente cuando las condiciones cambien, ya que no se está aplicando un fundamento sólido de las prácticas. De igual forma se pueden hallar casos donde las fábricas han implementado un máximo nivel de práctica y aún así rendir pobremente. En este sentido, las fábricas necesitan considerar si han escogido las prácticas correctas para su situación y si las prácticas se vinculan apropiadamente con la estrategia y entre sí.

Precisamente, partiendo de dicho modelo de HPM, el presente estudio examina el 
uso de la estrategia de operaciones y la tecnología, al testar individualmente el impacto de ambas PAP (al usar algunos de sus aspectos) sobre el rendimiento de operaciones. Así pues, seguidamente se razonan los constructos y conceptos usados en este estudio (sección 2) y en la sección 3 se hace una revisión de la literatura, presentado algunas posibles relaciones desde el marco de referencia del presente estudio y proponiendo un modelo y describiendo las hipótesis respectivas. Después, (sección 4), se describe la metodología de investigación de este trabajo y se discuten los métodos de análisis. Finalmente, en la sección 5 se resumen las conclusiones y consideraciones finales, resaltando las implicaciones y limitaciones del presente estudio.

\section{DEFINICIONESYCONCEPTOS}

En este estudio consideraremos y definiremos el rendimiento de operaciones, la estrategia de operaciones y la tecnología mediante algunos de los constructos definidos en HPM, ya que el foco de nuestro trabajo serán las relaciones entre ellas y, primordialmente, el vínculo entre las dos prácticas mencionadas y su posible impacto sobre el rendimiento.

Hay que recalcar que no proponemos las PAP y los constructos que aparecen en este estudio como "mejores prácticas" (paradigma por excelencia en la investigación sobre estrategia de operaciones en los ochenta y noventa (ej. Wheelwright y Bowen, 1996). Defendemos que las prácticas que se adoptan al imitar a fabricantes de clase mundial pueden contribuir a la paridad competitiva y no a la ventaja competitiva. Tales procesos no podrían y de hecho, debido a la contingencia (contexto), no pueden constituir una recomendación general para cualquier fábrica.

Además, el impacto competitivo debe considerarse debido a que la variable dependiente típica en un estudio de las relaciones entre PAP-rendimiento es algún tipo de rendimiento competitivo, ya sea financiero (Retorno Sobre el Activo (ROA, Return on Asset), Retorno Sobre la Inversión (ROI, Return on Investment), Retorno Sobre el Capital (ROE, Return on Equity)) o de operaciones (calidad, costo, etc.), en comparación con la competencia. Por ello, es obvio que las prácticas avanzadas de producción intensifican la competencia global entre fábricas y que, en la mayoría de las empresas, es necesaria una revisión de la estrategia de operaciones, pues cada práctica o combinación de ellas pueden ser apropiadas para diferentes entornos. Podrían conseguirse mejores rendimientos si las prestaciones clave de las PAP se analizan concienzudamente y sus conceptos se adoptan (o adaptan) e integran de forma cuidadosa, existiendo diferentes maneras de lograr los mismos resultados en 
distintos entornos (Sahin, 2000).

Finalmente, la selección de las dimensiones que se mostrarán a continuación no es exhaustiva ni es la única. Además, estas dimensiones no son exclusivas de HPM sino que son representativas de las PAP operando actualmente en las fábricas en general y sirven para los propósitos de presentar los argumentos teóricos.

\section{Rendimiento de operaciones}

Antes de discutir las implicaciones sobre el rendimiento de operaciones se debe definir y describir cuidadosamente su correspondiente constructo. Debido a que las fábricas productivas no controlan las medidas financieras de rendimiento, nuestra investigación se centrará en las medidas básicas que se controlan al nivel de la fábrica (ej. Miller y Roth, 1994; Cua et al., 2006), tales como costos, calidad, entrega y flexibilidad (ej. Hayes y Wheelwright, 1984; Ferdows y De Meyer, 1990). Además, la investigación internacional HPM hace referencia a otras medidas tales como rotación del inventario, tiempo de ciclo, nivel de servicio, innovación, etc. (ej. De Toni et al., 1992; Filippini etal., 1996).

Algunos autores piensan que las diferentes dimensiones del rendimiento de operaciones están positivamente correlacionadas entre sí (ej. Ferdows y De Meyer, 1990). Otros mantienen que estas dimensiones están sujetas a tradeoffs (incompatibilidades) y que, por tanto, están negativamente correlacionadas (ej. Skinner, 1969). Una forma de obtener una medida simple del rendimiento es modelarlo estratégicamente como un índice ponderado de las dimensiones señaladas. De esta manera, la medición del rendimiento incorpora las dimensiones relevantes del rendimiento de producción, pero al mismo tiempo pueden dar más peso a aquéllas que la dirección de la fábrica considera estratégicamente más importantes.

Por otro lado, Schroeder et al. (2002) consideran tres aspectos importantes en relación con el rendimiento. En primer lugar, las prioridades competitivas y las medidas del rendimiento varían tanto dentro de las industrias como entre ellas. En segundo lugar, una fábrica no puede lograr ventaja competitiva al concentrarse sólo en una dimensión de rendimiento, ya que la fábrica debe superar a la competencia en al menos una dimensión (ej. costo, calidad, entrega, flexibilidad, velocidad, etc.) y sobrepasar en el resto algún nivel mínimo (Ferdows y De Meyer, 1990; Hill, 1993; Wheelwright y Bowen, 1996). Por último, las fábricas buscan intencionadamente diferentes estrategias de operaciones compitiendo en diferentes dimensiones. 
Al considerar los múltiples aspectos del rendimiento es mejor ir más allá del uso de un modelo unidimensional 0 de una medida agregada estandarizada (estratégicamente ponderada) del rendimiento de operaciones (Schroeder et al., 2002). En términos prácticos, podemos utilizar una forma multidimensional de medidas estandarizadas de la industria (Schroeder y Flynn, 2001).

En nuestro caso, vamos a utilizar algunos de los índices del rendimiento competitivo de la fábrica productiva de acuerdo con el proyecto HPM (Schroeder y Flynn, 2001), tales como costo unitario de producción, calidad estándar del producto, entrega a tiempo, entrega rápida, flexibilidad para cambiar el mix de productos, flexibilidad para cambiar el volumen, tiempo del ciclo, velocidad de introducción de nuevos productos (tiempo de ejecución/lead time de desarrollo), innovación de productos, etc.

Estos índices representan distintas dimensiones de los objetivos básicos de la función de producción mencionados anteriormente (Skinner, 1969; Ferdows y De Meyer, 1990). Es decir, dichas dimensiones pueden coincidir con el conjunto de prioridades competitivas (costo, calidad, entrega, flexibilidad, innovación, etc.). Las dimensiones pueden medirse desde dos perspectivas, interna y externa. La perspectiva interna representa dimensiones que son útiles para el control y gestión interna del proceso de producción, mientras que la perspectiva externa implica dimensiones evaluadas por los clientes (Tabla 1).

\section{Tabla 1. Algunas medidas de rendimiento de operaciones}

(A partir del proyecto internacional HPM)

\begin{tabular}{|l|l|l|}
\hline $\begin{array}{c}\text { Dimensión de rendimiento } \\
\text { de operaciones }\end{array}$ & $\begin{array}{c}\text { Medidas de } \\
\text { rendimiento interna }\end{array}$ & \multicolumn{1}{|c|}{$\begin{array}{c}\text { Medidas de } \\
\text { rendimiento externa }\end{array}$} \\
\hline Costo & $\begin{array}{l}\text { Costo unitario de } \\
\text { producción }\end{array}$ & $\begin{array}{l}\text { Precio de mercado, precio } \\
\text { de venta del producto }\end{array}$ \\
\hline Calidad & $\begin{array}{l}\text { costos de control de } \\
\text { calidad, costos de } \\
\text { reproceso }\end{array}$ & $\begin{array}{l}\text { Productos ajustados a las } \\
\text { especificaciones }\end{array}$ \\
\hline Entrega & $\begin{array}{l}\text { Tiempo de ejecución } \\
\text { de producción }\end{array}$ & $\begin{array}{l}\text { Entrega a tiempo, tiempo de } \\
\text { ciclo, entrega rápida }\end{array}$ \\
\hline Flexibilidad & $\begin{array}{l}\text { Tiempo de } \\
\text { preparación }\end{array}$ & $\begin{array}{l}\text { Flexibilidad para cambiar el } \\
\text { mix de productos, flexibilidad } \\
\text { para cambiar el volúmen }\end{array}$ \\
\hline
\end{tabular}




\section{Estrategia de operaciones}

Cada vez más compañías reconocen la función de producción como una fuente potencial para conseguir una ventaja competitiva y como una manera de diferenciarse de los competidores. La necesidad de una orientación estratégica de la función de producción señala como factor crítico la definición e implementación clara de la estrategia de operaciones para determinar la eficacia de las fábricas productivas. Con todo, consideramos que no existe mucha investigación empírica documentada en la literatura de Dirección de Operaciones (y mucho menos en la investigación internacional de HPM) que trate, con una amplia base empírica, el impacto de la estrategia de operaciones sobre el rendimiento de la fábrica.

Tomando el esquema clásico definido en la literatura de estrategia que distingue entre procesos y contenidos (ej. Swamidass y Newell, 1987; Dangayach y Deshmukh, 2001), podemos decir que el proceso es clave para la formulación de la estrategia de operaciones. Éste debe ser un proceso formal de planificación estratégica donde la Dirección de la fábrica debe involucrarse por su conocimiento detallado de las capacidades de la fábrica. Además, la Dirección puede contribuir a la formulación de un plan a largo plazo que alinee exitosamente la estrategia de operaciones con la estrategia empresarial.

Una vez que se formula la estrategia de operaciones, ésta debe ser comunicada al personal de la fábrica para que entienda cómo las políticas y los objetivos proceden de ésta y para que puedan usarla como guía en la toma de decisiones. Con ello, se asume que la meta de la estrategia de operaciones es capacitar a la función de producción para proporcionar un apoyo aceptable a la estrategia empresarial. Consecuentemente, una estrategia de operaciones bien implementada y alineada dentro de una fábrica debe contener dimensiones tales como la previsión de nuevas tecnologías, el vínculo de la estrategia de operaciones con la estrategia empresarial, un proceso formal de planificación estratégica que involucre a la Dirección de la fábrica, la comunicación de la estrategia de operaciones al personal de fábrica y una estrategia fuerte o influyente dentro de la fábrica.

Desde el punto de vista de integración funcional/inderdepartamental del entorno de HPM, la estrategia de operaciones es una declaración de cómo el área de producción apoya a los objetivos generales de la empresa a través del diseño apropiado y de la utilización de recursos y capacidades de producción. En dicho apoyo es esencial el alineamiento de la estrategia de operaciones con la estrategia de marketing y con la estrategia de la empresa en general (Bates et al., 2001). Así 
pues, una estrategia de operaciones puede estar bien alineada e implementada si exhibe todas o casi todas las dimensiones descritas en este subapartado (Bates et al., 1995; 2001). Ello puede verse reflejado en la relación positiva entre la estrategia de operaciones y el rendimiento.

\section{Tecnología}

Existe una tendencia general de las fábricas productivas hacia el aumento en el empleo de tecnologías, debido a la hipótesis de que su utilización provocará mejoras de algunas medidas del rendimiento (ej.: reducción de costos o de recursos humanos, mejora de la calidad o de la flexibilidad). Desafortunadamente, en muchos casos, estas inversiones son criticadas por no producir los resultados deseados. Para comprender este hecho hay que tener en cuenta que la interconexión entre la tecnología y el rendimiento se ve influenciada por un conjunto de factores, algunos controlables y otros incontrolables, que son importantes en el resultado final.

Cuando se habla de tecnología es importante considerar no sólo la tecnología de productos (llamada también de desarrollo de nuevos productos) y la de procesos, sino también la de información y comunicaciones (TIC). Solamente cuando se integran estos tres tipos de tecnología y se ajustan a la fábrica y a sus prácticas puede haber una mejor adaptación tecnológica en el camino al alto rendimiento (Maier y Schroeder, 2001). Además, en el camino al alto rendimiento, la mera aplicación del enfoque de integración de tecnología es insuficiente, ya que dentro del HPM la tecnología no es un fin en sí misma.

Si una fábrica presenta una alta aplicación de todas las dimensiones conceptuales de tecnología de productos y de procesos, puede decirse que tiene un gran énfasis en tecnología y una visión progresiva hacia el desarrollo de tecnologías en las fábricas productivas. En la investigación HPM a nivel internacional se asume una definición abierta de tecnología, compuesta no sólo por los sistemas de hardware, sino también por los aspectos humanos y organizacionales empleados en la operativa de la empresa.

\section{Otros factores: variables de control}

Son muchos los factores que influyen en el rendimiento de una fábrica. Además de la estrategia de operaciones y de la tecnología, existen prácticas de infraestructura, otras PAP, múltiples factores del contexto, etc. La completa identificación de estos 
factores y su eliminación no es posible debido a las limitaciones de los datos. No obstante, basándonos en la revisión de la literatura de la investigación internacional de HPM, se identifican algunos factores como variables de control que pueden tener algún impacto sobre el rendimiento de la fábrica.

A pesar de ello, se espera que las PAP en cuestión permanezcan significativas después de la inclusión de las variables de control, dotando así de mayor robustez a los resultados obtenidos. Es decir, se defiende que las relaciones entre las PAP y el rendimiento, tal como se analiza en el presente estudio, son uniformemente aplicables a través de las variables de control.

\section{HIPÓTESIS DE TRABAJO Y PROPUESTAS}

Definitivamente lo que se hace afecta de forma concluyente al resultado. Así pues, el impacto de las prácticas avanzadas de producción sobre el rendimiento es una verdad absoluta y su comprobación empírica puede traer a luz deducciones interesantes. Así pues, resulta un desafío interesante investigar cómo determinada PAP es trascendental en el camino hacia el alto rendimiento. Por tanto, en nuestra investigación estudiamos dos tipos de relaciones: 1) que la variable tecnología por sí misma impacte a la variable de rendimiento y 2) que la variable estrategia de operaciones por sí misma impacte a la variable de rendimiento.

En relación con nuestro trabajo, el empleo de la noción de ajuste puede ser de utilidad. En general, el ajuste significa que la consistencia entre dos o más factores lleva a un mejor resultado (ver Cua et al., 2001; Milgrom y Roberts, 1995; Venkatraman y Prescott, 1990). Así pues, primero describiremos las propuestas del modelo de ajuste para las dos relaciones que estudiamos y presentaremos sus respectivas hipótesis. Para ello se propone un papel del efecto individual de cada PAP sobre el rendimiento desde la perspectiva del concepto de ajuste universal (Deleryy Doty, 1996; Ahmad et al, 2003), que se explica a continuación.

\section{Perspectiva Universal}

La perspectiva universal es la forma más simple de relación que puede existir entre dos variables. Esta perspectiva declara que el valor de una de las variables varía con cambios en el valor de la otra. Los investigadores han tratado de identificar "mejores prácticas" que tengan un efecto positivo sobre el rendimiento (ej. Delery and Doty 1996; Ahmad et al., 2003; Lee et al., 2004). Con esta perspectiva asumiremos que cualquiera de las dos prácticas seleccionadas (estrategia de operaciones o tecnología) se asocia positivamente al rendimiento de la fábrica (). 
Aunque sin profundizar, se consideran también factores contextuales (ej. tamaño de la fábrica, personalización de productos y utilización de la capacidad), como variables de control, que pueden afectar al rendimiento de producción; sin embargo, se espera que la implementación de las PAP resulte más explicativa de la variación del rendimiento.

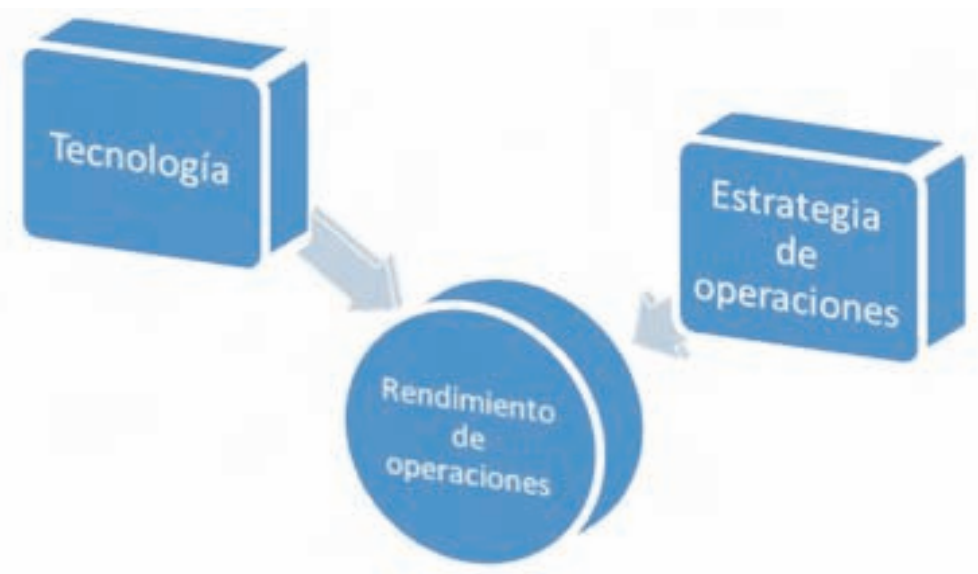

Figura . Perspectiva universal

\section{Relación Estrategia de Operaciones-Rendimiento de Operaciones}

La literatura de la investigación HPM a nivel internacional defiende que la estrategia de operaciones afecta positivamente al rendimiento de operaciones (ej. Bates et al., 2001; Milling et al., 1999). En la vinculación de la estrategia de operaciones con el rendimiento de operaciones se han creado varios enfoques. Hayes y sus coautores (Hayes y Wheelwright, 1984; Hayes, 1985; Hayes y Jaikumar, 1988; Hayes y Pisano, 1994) han argumentado constantemente que las capacidades de fabricación deben tener un papel importante en la competitividad de la empresa. Otros han demostrado un vínculo positivo entre la toma estratégica de decisiones y el rendimiento (Roth y Miller, 1990; Swamidass y Newell, 1987).

Demeter (2003) ha resumido trece estudios de algunos expertos que han revisado distintos elementos de la relación entre la estrategia de operaciones y el rendimiento de la empresa. Aunque los resultados obtenidos no son siempre concluyentes, parece existir vínculo en algún grado.

Por ello, y a pesar de que el cuerpo de investigación empírica en estrategia de 
operaciones, en general, no es muy grande, puede asumirse que existe apoyo empírico disponible para afirmar la existencia de dicho vínculo en algún grado. Por lo tanto, conforme a la, planteamos la hipótesis de que:

H1: La estrategia de operaciones se asocia positivamente con el rendimiento de operaciones.

Además, tomando variables contextuales planteamos que:

H2: La estrategia de operaciones mantiene su asociación positiva con el rendimiento de operaciones en presencia de factores contextuales.

\section{Relación Tecnología-Rendimiento de Operaciones}

De acuerdo con la literatura internacional de HPM, la tecnología puede mejorar el rendimiento de operaciones (Maier, 1998; Maier y Schroeder, 2001; Heine et al., 2003). Además, existe cierto apoyo empírico para esta afirmación: Bates y Flynn (1995) han encontrado altos rendimientos de producción por el uso de innovaciones tecnológicas; en los beneficios de los sistemas de información sobre la producción, Matsui y Sato (2001) y Bergeron et al. (1998) han revelado algunos resultados positivos; Mallick y Schroeder (2003) han estudiado empíricamente el rendimiento del desarrollo de productos. Por su parte, Heine et al. (2003) han revisado 16 modelos de la literatura del impacto de la tecnología sobre el rendimiento, proponiendo otro. Dichos modelos representan algunas perspectivas actuales sobre el papel de la tecnología y proveen una justificación razonable (empírica y teórica) de la relación entre tecnología y rendimiento.

Sin embargo, existe literatura que presenta apoyo empírico en sentido contrario, esto es, que la tecnología no influencia el rendimiento de la fábrica. Por ejemplo, Das y Jayaram (2003) resumen un total de 17 publicaciones en las que se sostiene que no existe una relación significativamente positiva entre la tecnología y el rendimiento. Es más, aún en la relación de la tecnología con la dimensión flexibilidad del rendimiento se encuentra evidencia contradictoria, que como Swink y Nair (2007) comentan es uno de los más comunes en la literatura de la relación tecnología-rendimiento. Al respecto ellos mencionan por un lado 7 publicaciones a favor y, por otro, 5 publicaciones en contra de dicha relación.

Por tanto, aunque la literatura empírica ha examinado la relación tecnologíarendimiento, los resultados no son aún definitivos, y menos aún respecto al rendimiento de operaciones, ya que la mayoría de dichos estudios analizan las 
relaciones desde la perspectiva del rendimiento de la empresa y no desde el rendimiento de operaciones.

Sin embargo, aunque los efectos de la tecnología sobre el rendimiento no están aún claros, planteamos la siguiente hipótesis, de acuerdo con el proyecto HPM internacional y la (más arriba), señalando que:

H3: La tecnología se asocia positivamente con el rendimiento de operaciones.

Y al igual que en la estrategia de operaciones, tomando variables contextuales, asumimos lo siguiente:

H4: La tecnología mantiene su asociación positiva con el rendimiento de operaciones en presencia de factores contextuales.

Para probar las hipótesis H1 y H3 se usa la ecuación 1 y para las hipótesis H2 y H4 se usa la ecuación 2 , donde $\mathrm{R}$ es el rendimiento, T representa la tecnología y $\mathrm{E}$ la estrategia de operaciones, además se introducen tres variables independientes como variables de control: TP es el In del tamaño de fábrica, PP es la personalización de productos, UC es la utilización de la capacidad de las fábricas ${ }^{1}$. Las â's son los coeficientes asociados a sus respectiva variables, mientras que åes el error. La significancia estadística del coeficiente â asociado con la variable de interés será la que provea, o no, apoyo para la perspectiva universal.

$$
R=\beta_{0}+\beta_{1} E+\beta_{2} T+\varepsilon
$$

$$
R=\beta_{0}+\beta_{1} E+\beta_{2} T+\beta_{3} T P+\beta_{4} P P+\beta_{5} U C+\varepsilon
$$

\section{SOBRE LA CONTRASTACIÓN DE HIPÓTESIS}

Las hipótesis mencionadas en el apartado anterior serán contrastadas mediante un survey a distintos sectores industriales de Honduras, aún por determinar. Los cuestionarios recogen aquellas preguntas que permiten dar respuesta a las

\footnotetext{
${ }^{1}$ Se pueden usar factores contextuales, definidos en el proyecto internacional HPM, tales como la utilización de la capacidad de la planta, la personalización del producto y el tamaño de la planta, entre otros.
} 
cuestiones de investigación planteadas no sólo de nuestro estudio sino de todo el contexto de HPM, potenciando el establecimiento de un cuerpo de conocimientos y el desarrollo de teorías, a través de la observación de fenómenos de la Dirección de Operaciones que no han sido empíricamente testados. Si bien es cierto que los cuestionarios habían de elaborarse a partir del análisis bibliográfico previo, hay que tener en cuenta que la investigación que presentamos es parte de un proyecto a nivel nacional que está enmarcado en una investigación internacional con varios años de funcionamiento, por lo que antes de iniciar nuestro estudio éstos ya habían sido previamente elaborados. No obstante, durante nuestro estudio los cuestionarios originales están siendo objeto de revisión por lo que respecta al estudio nacional para tener en cuenta las posibles influencias contextuales.

Las distintas escalas de medición y las cuestiones objetivas están ordenadas en un total de 12 cuestionarios dirigidos a otros tantos puestos dentro de cada fábrica, que usa 21 informantes en total. La mayoría de las escalas están incluidas en al menos dos cuestionarios diferentes, con objeto de poder triangular la información haciendo comparaciones entre grupos de encuestados (por ejemplo entre directivos y operarios de fábrica) y de minimizar la variabilidad derivada de las diferencias entre individuos, obteniendo así una mayor fiabilidad. Los ítems que conforman cada escala están mezclados (ordenados de diferente manera) en cada cuestionario, con idea de que no sea obvio qué ítem pertenece a cada escala o incluso que dichas escalas están siendo utilizadas. Una vez que las preguntas y las escalas fueron definidas, por el proyecto HPM a nivel internacional, se asignaron a los cuestionarios.

Por todo ello, a la hora de acometer un estudio HPM, por primera vez en Honduras con la presente investigación, nos encontramos con unos cuestionarios cuya fiabilidad y validez ha sido ampliamente contrastada, pero que no se han utilizado de forma inmediata. Dichos cuestionarios suman cientos de cuestiones, distribuidas en casi 1,500 preguntas, y los hemos revisado para observar si eran completamente adecuados al contexto industrial de nuestro país o si, por el contrario, requerían algún tipo de adaptación. Así pues, una vez traducidos se adaptarán en la medida de lo posible a los usos y terminologías de los sectores industriales parte del estudio HPM a nivel nacional.

Por lo que respecta a la traducción, el trabajo se repartió entre miembros del equipo investigador del proyecto de HPM en España (del cual es también miembro el autor del presente estudio), con objeto de que una excesiva dispersión no dificultara la mínima homogeneidad necesaria en las traducciones (recuérdese que muchos de los ítems recogidos en los diferentes cuestionarios implican idénticas preguntas a 
diferentes puestos).

Con los cuestionarios ya traducidos, será necesaria su adaptación a los sectores del estudio hondureño de HPM. Por ello, es importante reconocer diferencias en terminología entre los sectores y así lograr una interpretación consistente de cada pregunta cuando se entreviste a ejecutivos de un sector en particular. De lo contrario, algunas preguntas podrían generar datos de entrada no veraces 0 se puede correr el riesgo de obtener con excesiva frecuencia la respuesta "no aplicable". El resultado de dicha adaptación se revisará por todos los investigadores del equipo y potenciales entrevistadores, con objeto de asegurar la consistencia entre entrevistadores. Dado el alcance del estudio HPM hondureño (donde se enmarca la presente investigación), es importante que entre los entrevistadores se logre un grado de consistencia del enfoque. Por ello, desde el mismo momento en el que se construyeron los cuestionarios se enfatizó la necesidad de lograr un consenso en el protocolo de las entrevistas y en la interpretación de las preguntas. Finalmente, los cuestionarios se tradujeron de nuevo en ingles y se compararon con la versión inglesa original de los cuestionarios. Cualquier discrepancia encontrada se está corrigiendo antes de su empleo en Honduras.

Una futura investigación empírica permitirá incluir miles de datos específicos de un sinfín de fábricas hondureñas. Con objeto de conseguir la necesaria masa crítica de investigación para recoger estos datos en distintas regiones de Honduras, en el proyecto hondureño se están uniendo investigadores de varios grupos/centros de investigación de distintas universidades.

A continuación detallamos el método de encuestas y entrevistas, que se aplicarán a las fábricas de la muestra para lograr los objetivos propuestos en la recolección de datos.

Se estarán contactando fábricas de al menos 70 empleados procedentes de un muestreo. Se espera una relativa alta respuesta al contactar personalmente con las fábricas (usando en todos los casos los tres medios: teléfono, presentación y carta) y al prometerles que recibirán un perfil de fábrica para su comparación con el conjunto de las fábricas de su sector. Además, se está siendo uso de unas estrategias de alianza con distintas instituciones en Honduras, tanto privadas como públicas.

Además de los datos recabados con el cuestionario, se contemplará, cuando ello se juzgue necesario, la recogida de datos cualitativos adicionales mediante visitas guiadas a las fábricas de la muestra, así como a través de entrevistas estructuradas 
a los directores de fábrica, de recursos humanos, de calidad y de ingeniería de procesos. Las entrevistas sirven así de base para futuros casos de investigación y la consecuente construcción de teorías. Además, esta información adicional es muy útil para una interpretación más profunda y completa de los resultados obtenidos con el análisis de los datos cuantitativos.

\section{CONSIDERACIONES FINALES}

La presente investigación evalúa algunos factores empíricos críticos de éxito en el área de operaciones según los resultados de la investigación HPM en otros países. Con ello, se mostrarán aspectos críticos en los que el avance del conocimiento resulta especialmente necesario y urgente. En nuestra opinión, los resultados del presente estudio podrán contribuir de forma significativa en este sentido, como puede deducirse del contenido de los objetivos y la metodología.

Nuestro estudio lleva a cabo una recopilación, análisis y resumen exhaustivo de la literatura sobre el tema, basado en los aspectos teóricos de HPM. Entre los cimientos del modelo HPM se enfatiza, con la contingencia, la integración y la utilización de conceptos diferentes de dirección, para lograr la habilidad de una unidad de fabricación en alcanzar mejoras continuas en el área de producción. Este objetivo de HPM por lograr y mantener el alto rendimiento es evasivo debido a que cada fábrica es única (contingencia) y por tanto no puede haber un método universal para alcanzar y sostener el estatus HPM.

Como inicio y enlace al estudio de campo, se está preparando la muestra de fábricas productivas como unidad de estudio. Para su aplicación empírica en la industria, hemos mencionado algunas dimensiones que son parte de las escalas y medidas de HPM. Las últimas son de gran importancia como cimiento para la creación de los cuestionarios de acuerdo con el contexto de la industria hondureña. A pesar de que la muestra, que se estudiará empíricamente, pueda ser reducida, para el desarrollo de las escalas y medidas, el análisis estadístico planteado en todo momento por la investigación internacional HPM ha sido de naturaleza inferencial.

Uno de los puntos fuertes de este trabajo, en nuestra opinión, es el de los instrumentos de recogida de datos: los cuestionarios. Éstos han sido diseñados de manera que recogen resultados empíricos fiables y nacionalmente comparables, los cuales reflejan los aspectos multifacéticos de las modernas estructuras de producción, ya que condicionarán las conclusiones relevantes que se puedan derivar. Estos resultados del estudio de campo permitirán a las compañías 
involucradas evaluar diferencias con el conjunto de todas las compañías de su industria y eventualmente analizar debilidades existentes y aumentar potenciales existentes. Asimismo, es necesario tener siempre presente la posibilidad de una futura comparación nacional, con la importancia particular de verificar si existen diferencias entre las compañías HP y el resto. Esto no significa modificar la metodología seguida, ya que el modo de recopilación de respuestas se ajusta a muestras grandes y áreas geográficas extensas. Por otro lado, a pesar de que se propone una construcción de cuestionarios extensos, las medidas que se presentan aseguran la participación de la población o muestra.

La extensión y exhaustividad de los cuestionarios permiten la recopilación de datos objetivos y opiniones subjetivas de las fábricas que participan. Estos datos y opiniones están siendo registrados en bancos de datos regionales y nacionales. Debido a esta consolidación de datos extensos y consistentes, es posible el análisis nacional de factores de éxito en el área de la producción. Con esto se conseguirá que en el estudio empírico se analicen en detalle cómo las áreas de producción de compañías exitosas (HP) difieren de las menos exitosas.

Finalmente, el objetivo principal planteado en el presente trabajo es un estudio de campo sobre la aplicación de HPM que investiga la caracterización de las PAP, estrategia de operaciones y tecnología, y sus impactos sobre el rendimiento. Así pues, con la perspectiva universal proponemos examinar si la estrategia de operaciones o/y tecnología son eficaces por sí mismas para mejorar el rendimiento de las operaciones.

Por lo que el estudio de campo es una aportación apreciable, ya que se espera encontrar el uso (consciente o no, parcial o total) de algunos de los fundamentos de HPM por parte de algunas fábricas.

Por otra parte, las lecciones aprendidas en la investigación se pueden propagar a la industria en general por medio de la difusión de los resultados del estudio de campo, así como a través de medios electrónicos.

\section{Limitaciones del estudio y desarrollo futuro de la investigación}

El modelo universal impone una correspondencia lineal entre la estrategia de operaciones o la tecnología y sus respectivos resultados directos con el rendimiento. Esta forma de ajuste no toma en consideración relaciones más complejas tales como las interrelaciones entre las PAP. Sin embargo, esta 
limitación puede superarse por un futuro estudio que pueda extender el actual desde otra perspectiva, tal como un ajuste de selección o de interacción, que contemple hipótesis de dependencias entre el ajuste de ambas PAP y el vínculo entre dicho ajuste y rendimiento. Ello permitirá la pregunta de si el resultado se mejora por la interacción entre ambas PAP.

\section{RECONOCIMIENTO}

El presente trabajo tiene como marco base el proyecto HPM de investigación científica y desarrollo tecnológico del Programa Nacional de Diseño de Producción Industrial del Ministerio de Educación y Ciencia de España (DPI 2006).

\section{BIBLIOGRAFÍA}

Ahmad, S., Schroeder, R. and Sinha, K. (2003). "The role of infrastructure practices in the effectiveness of JIT practices: implications for plant competitiveness". Journal of Engineering and Technology Management. 20:161-191.

Bates, K, Blackmon, K., Flynn, E. and Voss C. (2001). "Manufacturing Strategy: Building Capability for Dynamic Markets". High Performance ManufacturingGlobal Perspectives. New York: John Wiley \& Sons, Inc. p. 42-72.

Bates, K., S. Amundson, W. Morris and Schroeder, R. (1995). "The Crucial Interrelationship between Manufacturing Strategy and Organizational Culture". Management Science. 41(10).

Bergeron, F., Raymond, L., Gladu, M. and Leclerc, D. (1998). "The contribution of information technology to the performance of SMEs: alignment of critical dimensions". Proceedings of the European Conference on Information Systems, Aix-en Provence. p.173-187.

Cua, K., McKone, K. and Schroeder, R. (2001). "Relationships between implementation of TQM, JIT, and TPM and manufacturing performance". Journal of Operations Management. 19: 675-694.

Cua, K., McKone-Sweet, K. and Schroeder, R. (2006). "Improving Performance through an Integrated Manufacturing Program". The Quality Management Journal. 13 (3): 45.

Dangayach, G and Deshmukh, S. (2001). "Manufacturing strategy. Literature review and some issues". International Journal of Operations \& Production Management. 21 (7): 884-932.

Das, A. and Jayaram, J. (2003). "Relative importance of contingency variables for advanced manufacturing technology". International Journal of Production Research. 41(18): 4429-4452.

De Toni, A., Filippini, R. and Forza, C. (1992). "Manufacturing Strategy in Global 
Markets: An Operations Management Model". International Journal of Operations \& Productions Management. 12 (4): 7-18.

Delery, J. and Doty, D. (1996). "Modes of theorizing in strategic human resource management: Tests of universalistic, contingency, and configurational performance predictions". Academy Management Journal. 39(4) 802-835.

Demeter K. (2003). "Manufacturing strategy y competitiveness". International Journal of Production Economics. 81-82, 205-213.

Ferdows, K. and De Meyer, A. (1990). "Lasting Improvements in Manufacturing Performance: In Search of New Theory". Journal of Operations Management. . 9 (2).

Filippini, R., Forza, C. and Vinelli, A. (1996). "Improvement initiative paths in operations". Integrated Manufacturing Systems, 7 (2): 67-76.

Hayes R. H. (1985): "Strategic planning - forward in reverse?". Harvard Business Review. 63:67-77.

Hayes R. H. and Jaikumar R. (1988): "Manufacturing's crisis: New technologies, obsolete organizations". Harvard Business Review. 66: 77-85.

Hayes R. H. and Pisano G. P. (1994): "Beyond world-class: The new manufacturing strategy". Harvard Business Review. 72: 77-84.

Hayes, R.H. and Wheelwright S.C. (1984). Restoring Our Competitive Edge. New York: Free Press.

Heine, M. L., Grover, V. and Malhotrac, M.K. (2003). "The relationship between technology and performance: a meta-analysis of technology models". Omega, 31: 189-204.

Hill T. (1993). The essence of operations management. New York. Prentice Hall.

Lee, J., Miranda, S. and Kim, AND. (2004). "IT Outsourcing Strategies: Universalistic, Contingency, and Configurational Explanations of Success". Information Systems Research. 15 (2) 110-131.

Maier, F. and Schroeder, R. (2001). "Competitive Product and Process Technology". En: Schroeder and Flynn (dir), High Performance Manufacturing-Global Perspectives. New York: John Wiley \& Sons, Inc. p. 74-114.

Mallick, D and Schroeder, R. (2005). "An Integrated Framework for Measuring Product Development Performance in High Technology Industries". Production and Operations Management (POM). 14 (2) 142-158.

Matsui, A. and Sato, O. (2001). "A Comparative Analysis on the Benefits of Production Information Systems," San Francisco, USA: Proceedings of the 32nd Annual Meeting of Decision Sciences Institute. p.687-689.

Milgrom, P. and Roberts, J. (1995). "Complementarities and fit: strategy, structure and organizational change in manufacturing". Journal of Accounting and Economics. 19 (2-3): 179-208.

Milling, P., Maier, F. and Mansury, D. (1999): "Impact of manufacturing strategy on 
plant performance-insights from the international research project: world class manufacturing". In: Emilio Bartezzaghi et al. Managing Operations Networks. p. 573-580.

Milling, P., Maier, F. y Mansury, D. (1999): "Impact of manufacturing strategy on plant performance-insights from the international research project: world class manufacturing". In: Emilio Bartezzaghi, Roberto Fillipini, Gianluca Spina und Andrea Vinelli (Eds.): Managing Operations Networks - European Operations Management Association Conference. Venice, Italy. p. 573 - 580.

Roth A. V. and Miller J. G. (1990). "Manufacturing strategy, manufacturing strength, managerial success and economic outcomes". En: Ettlie, J.G., Burstein, M. C. and A. Feigenbaum (dir), Manufacturing Strategy: The research agenda for the next decade. Boston, MA: Kluwer. 97-109.

Sahin, F. (2000). "Manufacturing competitiveness: Different systems to achieve the same results". Production and Inventory Management Journal. First Quarter. $41(1): 56$.

Schroeder, R. G. and Flynn, B., Editors (2001). High Performance ManufacturingGlobal Perspectives. New York: John Wiley \& Sons, Inc.

Schroeder, R., Bates K. and Junttila, M. (2002). "A resource-based view of manufacturing strategy and the relationship to manufacturing performance". Strategic Management Journal. 23: 105-117.

Skinner, W. (1969): "Manufacturing - Missing link in corporate strategy". Harvard Business Review. May-June. 136-145.

Swamidass, P. and Newell, T. (1987). "Manufacturing strategy, environmental uncertainty and performance: A path analytic model". Management Science. 33 (4).

Swink, M. and Nair, A. (2007). "Capturing the competitive advantages of AMT: Design-manufacturing integration as a complementary asset". Journal of Operations Management. 25: 736-754.

Venkatraman, N. and Prescott, J.E. (1990). "Environment-strategy coalignment: an empirical test of its performance implications". Strategic Management Journal. 11 (1): 1-23.

Wheelwright S. C. and Bowen H. (1996): "The Challenge of Manufacturing Advantage". Production and Operations Management. 5: 59-77. 